\title{
CONSTRUCTING NEAR-HADAMARD DESIGNS WITH (ALMOST) D-OPTIMALITY BY GENERAL SUPPLEMENTARY DIFFERENCE SETS
}

\author{
Yuan-Lung Lin and Frederick Kin Hing Phoa \\ Academia Sinica
}

\begin{abstract}
We propose a new and unified construction method, general supplementary difference sets (GSDS)s, for near-Hadamard designs when the run sizes are $n \equiv 2(\bmod 4)$. These designs possess high $D$-efficiencies. Eihlich (1964) derived an upper bound for the determinant of matrices of order $n \equiv 2(\bmod 4)$ achievable only if $2 n-2$ is a sum of two squares. Between 1 to 100, there are 6 parameters, $22,34,58,70,78$, and 94 , that do not fulfill this condition. We formulate a class of near-Hadamard designs whose determinants are very close to Ehlich's upper bound, and construct these designs for many values of $n$.
\end{abstract}

Key words and phrases: Difference sets, general supplementary difference sets, Hadamard design, near-Hadamard design, optimal designs.

\section{Introduction}

There is much interest in studying large-scale systems and considerable effort goes into designing more efficient studies. Careful design considerations, even with only minor variations from traditional designs, can provide more precise estimates or ability to estimate more effects at the same cost (Phoa, Wong, and Xul (200.9); Phoa, Xu, and Wong (200.9); Xu, Phoa, and Wong (200.9)).

We propose a systematic approach to constructing a class of two-level square designs with large determinant. To quantify the $D$-optimality of a design, we adopt the $D$-efficiency criterion of tones and Nachtsheim (20II) and Phoa and Lin (2015):

$$
d_{e}\left(\boldsymbol{D}, \boldsymbol{D}_{o}\right)=\left(\frac{\left|X(\boldsymbol{D})^{\prime} X(\boldsymbol{D})\right|}{\left|X\left(\boldsymbol{D}_{o}\right)^{\prime} X\left(\boldsymbol{D}_{o}\right)\right|}\right)^{1 / p}
$$

where $X(\boldsymbol{D})$ and $X\left(\boldsymbol{D}_{o}\right)$ are the design matrices of design $\boldsymbol{D}$ and $D$-optimal design $\boldsymbol{D}_{o}$ respectively, $|\boldsymbol{M}|$ is the determinant of a matrix $\boldsymbol{M}$, and $p$ is the number of terms in the model that consists of all main effects.

For notation here, $\boldsymbol{I}_{n}$ denotes an identity matrix of order $n, \boldsymbol{J}_{n}$ an $n \times n$ square matrix with all entries +1 , and $\boldsymbol{j}$ an $n \times 1$ all-ones vector; $\boldsymbol{A}^{\prime}$ denotes the transpose of a matrix $\boldsymbol{A} ;+$ and - are used as abbreviations for +1 and $-1 ;|\boldsymbol{D}|$ 
or $\operatorname{det}(\boldsymbol{D})$ denotes the determinant of a matrix $\boldsymbol{D}$, and $|c|$ denotes the absolute value of a constant $c$.

Let $\mathcal{X}_{n}$ be the set of all \pm 1 designs of order $n$. Hadamard (18.93) proved that for all $\boldsymbol{X}=\left(x_{i, j}\right) \in \mathcal{X}_{n}$

$$
\operatorname{det}(\boldsymbol{X}) \leq\left(\prod_{i=1}^{n} \sum_{j=1}^{n} x_{i, j}^{2}\right)^{1 / 2} \leq n^{n / 2}
$$

And if $\left|x_{i, j}\right| \leq 1$ and the equality is satisfied, then the design $\boldsymbol{X}$ is ( \pm 1$)$ valued and $n$ is 1,2 , or a multiple of 4 .

A D-optimal design of order $n$ is an $n \times n$ matrix with entries \pm 1 having maximum determinant. For $n \equiv 2(\bmod 4)$, say $n=2 v$, Ehlich (1964), and independently Wojtas ([1564), proved that for all $\boldsymbol{X} \in \mathcal{X}_{n}$,

$$
\operatorname{det}(\boldsymbol{X}) \leq(2 n-2)(n-2)^{(n / 2)-1}
$$

A necessary condition for equality in $(\mathbb{L}$. $)$ to hold is that $2 n-2$ (equivalently $n-1$ ) is a sum of two squares, see Ehlich (1964); we refer to the upper bound as Ehlich's upper bound. Cohn ([1989) proved that equality holds if and only if there exists $\boldsymbol{X} \in \mathcal{X}_{n}$ such that

$$
\boldsymbol{X} \boldsymbol{X}^{\prime}=\boldsymbol{X}^{\prime} \boldsymbol{X}=\boldsymbol{L} \otimes \boldsymbol{I}_{2}
$$

where $\boldsymbol{L}=(n-2) \boldsymbol{I}+2 \boldsymbol{J}$ is a $v \times v$ matrix and $\otimes$ is the Kronecker product. Ehlich (1564) also proved that if $\boldsymbol{A}$ and $\boldsymbol{B}$ are commuting \pm 1 matrices of order $v$ such that

$$
\boldsymbol{A} \boldsymbol{A}^{\prime}+\boldsymbol{B} \boldsymbol{B}^{\prime}=2(v-1) \boldsymbol{I}_{v}+2 \boldsymbol{J}_{v}
$$

then the matrix

$$
\boldsymbol{X}=\left(\begin{array}{rr}
\boldsymbol{A} & \boldsymbol{B} \\
-\boldsymbol{B}^{\prime} & \boldsymbol{A}^{\prime}
\end{array}\right)
$$

has the maximum determinant. Such $\boldsymbol{A}$ and $\boldsymbol{B}$ can be constructed by using supplementary difference sets (SDSs) with parameters $(v ; r, s ; \lambda)$ (Wallis (एप, 2 )).

There are two known infinite families of $D$-optimal designs, one for $v=$ $q^{2}+q+1$, where $q$ is a prime power, and one for $v=2 q^{2}+2 q+1$, where $q$ is an odd prime power (Koukouvinos, Kounias, and Seberry (1991); Whiteman (19.90)). More details are given by Colbourn (2य10), Đoković (ए1997), and Gysin and Seberry (1098). Đoković and Kotsireas (2012) give a comprehensive table of all odd $v<200$ for which $D$-optimal SDS are known, but there are still many unknown cases. For $n=22,34,58,70,78,94(n \leq 100)$ Ehlich's upper bound cannot be attained as $2 n-2$ is not a sum of two squares. Orrick and Slolmon 
(20102) created a website (http://www.indiana.edu/ maxdet) which lists an $n \times n$ design with the largest known determinant for $1 \leq n \leq 119$.

In Section 2, we introduce general supplementary difference sets (GSDS)s. In Section 3, we define near-Hadamard designs and evaluate their determinants. We utilize GSDSs as a unified method for constructing near-Hadamard designs that are almost $D$-optimal. For $n=22,34,58,70,78,94$, these designs have at least 99.5\% $D$-efficiency. When $n \equiv 0(\bmod 4)$, assuming the Hadamard conjecture is true, a $D$-optimal design is called a Hadamard design of order $n$. If $\boldsymbol{H}$ is a Hadamard design of order $n$, then the rows in $\boldsymbol{H}$ are pairwise orthogonal, $\boldsymbol{H} \boldsymbol{H}^{T}=n \boldsymbol{I}_{n}$, and the Hadamard conjecture says that such designs with \pm 1 entries exist if $n$ is divisible by 4 . Hadamard designs were originally investigated as a family of orthogonal designs by Sylvester (1867), he constructed Hadamard designs of orders power of two. Fletcher, Gysin, and Seberry (2001) constructed Hadamard designs with two circulant cores. These designs are obtained by using SDSs. Kotsireas, Koukouvinos, and Seberry (2006) conjectured that Hadamard designs of order $n \geq 8$ can be constructed via SDSs in a systematic and unified way. In this paper, we utilize GSDSs to construct near-Hadamard designs and formulate two types of near-Hadamard designs of order $n$. If it exists, each type is conjectured to be $D$-optimal when $2 n-2$ is not a sum of two squares.

\section{Preliminaries and Definitions}

Difference sets are powerful tools for constructing balanced incomplete block designs (BIBD). For example, Bose (II939) used pure and mixed difference sets to construct some special BIBDs. Many examples and results about difference sets can be found in Andersen (1990) and Wallis (2007). An $n \times n$ matrix $\boldsymbol{A}=\left(a_{i, j}\right)$ is circulant if $a_{i+1, j+1}=a_{i, j}$ where the subscripts are reduced modulo $n$. Such a circulant matrix $\boldsymbol{A}$ can be obtained from its first row, so it can be denoted by $\boldsymbol{A}=\operatorname{circ}\left(a_{1,1}, a_{1,2}, \ldots, a_{1, n}\right)$. Suppose that $\mathcal{G}$ is an Abelian group of order $v$, written in additive notation, and suppose $\mathcal{B}$ is a set of $k$ elements of $\mathcal{G}$. Then the design generated from $\mathcal{B}$ (in $\mathcal{G}$ ) consists of all the blocks $\{\mathcal{B}+g: g \in \mathcal{G}\}$, where $\mathcal{B}+g=\{x+g:$ for all $x \in \mathcal{B}\}$. It is a symmetric block design, and $\mathcal{B}$ is called the base block. We only consider the Abelian group $Z_{v}$. The concept of general difference set (GDS) was proposed by Lin, Phoa, and KaO (2015).

Definition 1. A $\left(v, k ; \lambda_{1}, \ldots, \lambda_{\lfloor v / 2\rfloor}\right)$ GDS is a set $D=\left\{d_{1}, \ldots, d_{k}\right\}$ of distinct elements of $Z_{v}$ such that each difference $( \pm i)$ appears $\lambda_{i}$ times for all $i=1, \ldots,\lfloor v / 2\rfloor$.

If $\lambda_{1}=\ldots=\lambda_{\lfloor v / 2\rfloor}=\lambda$, we write $(v, k, \lambda)$ for short. Difference sets are the special cases of GDSs, where a GDS allows each difference to appear different times. In general, a GDS can be represented by its incidence matrix. 
Table 1. The incidence matrix $\boldsymbol{D}$.

\begin{tabular}{|ccccccccc|}
\hline & 1 & 2 & 3 & 4 & 5 & 6 & 7 & 8 \\
$D+0$ & - & - & - & + & + & + & + & + \\
$D+1$ & + & - & - & - & + & + & + & + \\
$D+2$ & + & + & - & - & - & + & + & + \\
$D+3$ & + & + & + & - & - & - & + & + \\
$D+4$ & + & + & + & + & - & - & - & + \\
$D+5$ & + & + & + & + & + & - & - & - \\
$D+6$ & - & + & + & + & + & + & - & - \\
$D+7$ & - & - & + & + & + & + & + & - \\
\hline
\end{tabular}

Definition 2. Let $D$ be a $\left(v, k ; \lambda_{1}, \ldots, \lambda_{\lfloor v / 2\rfloor}\right)$ GDS. The incidence matrix of $D$ is a $v \times v$ matrix $\boldsymbol{D}$ with entries

$$
d_{i, j}= \begin{cases}- & \text { if } j \in D+(i-1) \\ + & \text { otherwise }\end{cases}
$$

$\boldsymbol{D}$ is a circulant matrix, where the first row of $\boldsymbol{D}$ corresponds to the base block $D$ and each row of $\boldsymbol{D}$ is a right shift of the previous row.

Example 1. Choose elements 1,2, and 3 in $Z_{8}$ to form a set $D$. The differences \pm 1 and \pm 2 appear exactly twice and once respectively, so $D$ is a $(8,3 ; 2,1,0,0)$ GDS. By definition the first row of the incidence matrix $\boldsymbol{D}$ is obtained by placing " - " at the position $i$ if $i \in D$ and "+" otherwise (see Table 1).

For special parameters, such an incidence matrix directly constructs Hadamard designs.

Example 2. If $D=\{2,4,5,6,10\}$, then $D$ is a $(11,5,2)$ difference set. The incidence matrix $\boldsymbol{D}$ is an $11 \times 11$ design. By adding a row with all "-" entries, we obtain the Plackett and Burman design of 11 factors and 12 runs. A further addition of a column with all "+" entries leads to a Hadamard design of order 12 .

Hall and Ryser (195I) proved the multiplier theorem as a necessary condition for the existence of a $(v, k, \lambda)$ difference set. For example, it is impossible to find a $(37,9,2)$ difference set (Andersen (11990) ). Since a difference set may not exist for a given set of parameters, we propose general supplementary difference sets (GSDS).

Let $D_{1}, \ldots, D_{n}$ be subsets of $Z_{v}$ (or any finite abelian group of order $v$ ) containing $k_{1}, \ldots, k_{n}$ elements respectively. Let $T_{i}$ be the union of all differences between elements of $D_{i}$ (with repetitions), and let $T$ be the union of all the elements of the $T_{i}$. 
Definition 3. If $T$ contains each non-zero element of $Z_{v} \lambda_{j}$ times for $j=$ $1, \ldots,\lfloor v / 2\rfloor$, then the sets are called $n-\left\{v ; k_{1}, \ldots, k_{n} ; \lambda_{1}, \ldots, \lambda_{\lfloor v / 2\rfloor}\right\}$ general supplementary difference set (GSDS).

We let $n(\lambda)$ be the number of the differences $g \in Z_{v}$ that appears $\lambda$ times in such a $T$. Sometimes we write down distinct $\lambda$ only for short. An SDS is a special case of GSDS where each difference appears an equal number of times (Wallis ([प/2)). If $k_{1}=\ldots=k_{n}=k$, we write $n-\left\{v ; k ; \lambda_{1}, \ldots, \lambda_{\lfloor v / 2\rfloor}\right\}$ GSDS in brief. When $k_{i}=k$ and $\lambda_{j}=\lambda$ for all $i, j$, an $n-\{v ; k ; \lambda\} \operatorname{GSDS}$ is a $(v, k, \lambda)$ difference family (or difference system) with $n$ base blocks (Colbourn (2010)).

\section{Example 3.}

(a) The sets $\{1,2,4,6\}$ and $\{1,3,6\}$ are a $\{8,4 ; 1,2,2,1\}$ GDS and a $\{8,3 ; 0$, $1,2,0\}$ GDS, respectively. Hence, $\{1,2,4,6\}$ and $\{1,3,6\}$ form a $2-\{8 ; 4,3 ; 1$, $3,4,1\}$ GSDS. In this case, the number of the differences that appear once is 2 , so $n(1)=2$. It follows that $n(2)=0, n(3)=1$ and $n(4)=1$.

(b) $\{1,3,4,9,10,12\}$ and $\{2,5,6,7,8,11\}$ form a $2-\{13 ; 6 ; 5\}$ GSDS.

Obviously, two general difference sets together constitute a GSDS.

\section{Construction of Near-Hadamard Designs}

A $D$-optimal design of order $n \equiv 2(\bmod 4)$ achieving Ehlich's upper bound exists only if $2 n-2$ is a sum of two squares. Therefore, one can consider a different structure from the SDS to construct designs with high $D$-efficiencies when a $D$-optimal design achieving Ehlich's upper bound cannot exist. We use the $n \times n$ block matrix

$$
\boldsymbol{M}=\left(\begin{array}{rrcc}
c & c & \boldsymbol{j}^{\prime} & \boldsymbol{j}^{\prime} \\
c & -c & \boldsymbol{j}^{\prime} & -\boldsymbol{j}^{\prime} \\
\boldsymbol{j} & \boldsymbol{j} & \boldsymbol{A} & \boldsymbol{B} \\
\boldsymbol{j} & -\boldsymbol{j} & \boldsymbol{B}^{\prime} & -\boldsymbol{A}^{\prime}
\end{array}\right),
$$

where $|c|=1$ and $\boldsymbol{j}$ is the $(n-2) / 2 \times 1$ vector of ones, and take a near-Hadamard design to mean a design that does not reach Ehlich's upper bound, but has a very large determinant.

We set our two types of near-Hadamard designs. Let $n=2 v+2$ and $\boldsymbol{M}$ be an $n \times n$ matrix with \pm 1 entries such that

$$
\boldsymbol{M} \boldsymbol{M}^{\prime}=\left(\begin{array}{cccc}
n & 0 & c_{1} \boldsymbol{j}^{\prime} & 0 \\
0 & n & 0 & c_{2} \boldsymbol{j}^{\prime} \\
c_{1} \boldsymbol{j} & 0 & \boldsymbol{P} & 0 \\
0 & c_{2} \boldsymbol{j} & 0 & \boldsymbol{P}
\end{array}\right)
$$


where $\left|c_{1}\right|=\left|c_{2}\right|=2$ and $\boldsymbol{P}_{v \times v}=\operatorname{circ}\left(n, s_{2}, \ldots, s_{v}\right)$. If $\left|s_{i}\right|=2$ for all $i=$ $2,3, \ldots, v$, then $\boldsymbol{M}$ is called a near-Hadamard design; if $s_{i}=2(-1)^{i-1}$ for all $i=2,3, \ldots, v$, then $\boldsymbol{M}$ is called a near-Hadamard design of Type $I$, denoted by $M_{I}$; if $s_{(v / 2)+1}=2$ and $s_{i}=2(-1)^{i-1}$ for all $i=2,3, \ldots, v$ except $i=(v / 2)+1$, then $\boldsymbol{M}$ is called a near-Hadamard design of Type $I I$, denoted by $\boldsymbol{M}_{I I}$. The $\boldsymbol{P}$ matrices for the two types are denoted by $\boldsymbol{P}_{I}$ and $\boldsymbol{P}_{I I}$, respectively.

By Cohn's theorem, a $D$-optimal design $\boldsymbol{X}$ achieving Ehlich's upper bound must satisfy $(\mathbb{L} 2)$, so $\operatorname{det}(\boldsymbol{X})=[\operatorname{det}(\boldsymbol{L})]^{2}$. Cohn (ए989) found an upper bound for $\operatorname{det}(\boldsymbol{L})$.

Lemma 1. Let $k \geq 2, \boldsymbol{K}(k)$ denote a positive-definite symmetric matrix with diagonal elements $k_{r, r}=n$ and with $\left|k_{r, s}\right| \geq 2$. Then $\operatorname{det}(\boldsymbol{K}) \leq(n+2 k-2)(n-$ $2)^{k-1}$, and equality holds if and only if $\boldsymbol{K}=\Sigma \boldsymbol{L} \Sigma$ for $\Sigma$ some suitable square diagonal matrix with all its diagonal elements \pm 1 .

For even $k$ and $\alpha=(1,-1,1,-1, \ldots, 1,-1)$ a $k \times 1$ vector, $\operatorname{diag}(\alpha)$ is a $\Sigma$ such that the equality holds. Since $\boldsymbol{P}_{I}=\Sigma \boldsymbol{L} \Sigma, \operatorname{det}\left(\boldsymbol{P}_{I}\right)$ reaches the upper bound in Lemma 1, and $\boldsymbol{M}_{I}$ has the maximum determinant among all $\boldsymbol{M}$ that have the form (B.]). Because $\boldsymbol{P}_{I}$ may not exist for some $n, \boldsymbol{P}_{I I}$ is considered though $\operatorname{det}\left(\boldsymbol{P}_{I I}\right)$ is smaller than $\operatorname{det}\left(\boldsymbol{P}_{I}\right)$. If $\boldsymbol{P}_{I}$ and $\boldsymbol{P}_{I I}$ do not exist, then a general $\boldsymbol{P}$ matrix is another choice. Since the total number of \pm 2 's in matrix (ए2) and $\boldsymbol{M} \boldsymbol{M}^{\prime}$ are the same, a general near-Hadamard design still has high $D$-efficiency.

Lemma 2. Let $\boldsymbol{M}$ be a near-Hadamard design of order $n \equiv 2(\bmod 4)$. Then $\operatorname{det}\left(\boldsymbol{M}_{I}\right)=2(n-2)^{n / 2}$, and $\operatorname{det}\left(\boldsymbol{M}_{I I}\right)=2(n-4)\left(n^{2}+4\right)(n-6)^{(n-6) / 4}(n+$ $2)^{(n-6) / 4}$.

Since $\boldsymbol{P}_{I}=\Sigma \boldsymbol{L} \Sigma$ for a suitable $\Sigma$, its determinant should be maximum among all matrices satisfying the conditions of Lemma 1 . The difference between $\boldsymbol{P}_{I}$ and $\boldsymbol{P}_{I I}$ is the sign of $s_{(v / 2)+1}$. By Lemma $2, \operatorname{det}\left(\boldsymbol{M}_{I I}\right)$ is smaller than $\operatorname{det}\left(\boldsymbol{M}_{I}\right)$. Moreover, $\boldsymbol{M}_{I}$ has $D$-efficiency $((n-2) /(n-1))^{2 / n}$.

Example 4. Let $\boldsymbol{A}=\operatorname{circ}(-,-,+,-,+,-,+,-,+,+), \boldsymbol{B}=\operatorname{circ}(-,-,-,-,+$, $+,-,+,+,+)$ and $\boldsymbol{M}$ be a matrix of order 22 , as follows (next page).

This is a near-Hadamard design of Type $I$ with at least $99.56 \% D$-efficiency. The submatrices $\boldsymbol{A}$ and $\boldsymbol{B}$ are constructed by GSDS.

A $(v, k, \lambda)$ difference set is equivalent to a symmetric BIBD with parameter $(v, b, r, k, \lambda)$, where $b$ is the number of blocks and each element appears in $r$ blocks. If $\boldsymbol{A}$ is the incidence matrix of a $(v, b, r, k, \lambda)$ BIBD, then $\boldsymbol{A A}^{\prime}=(r-$ $\lambda) \boldsymbol{I}_{v}+\lambda \boldsymbol{J}_{v}$. Lin, Phoa, and KaO (2015) proved the following regarding incidence matrices of GDSs. 


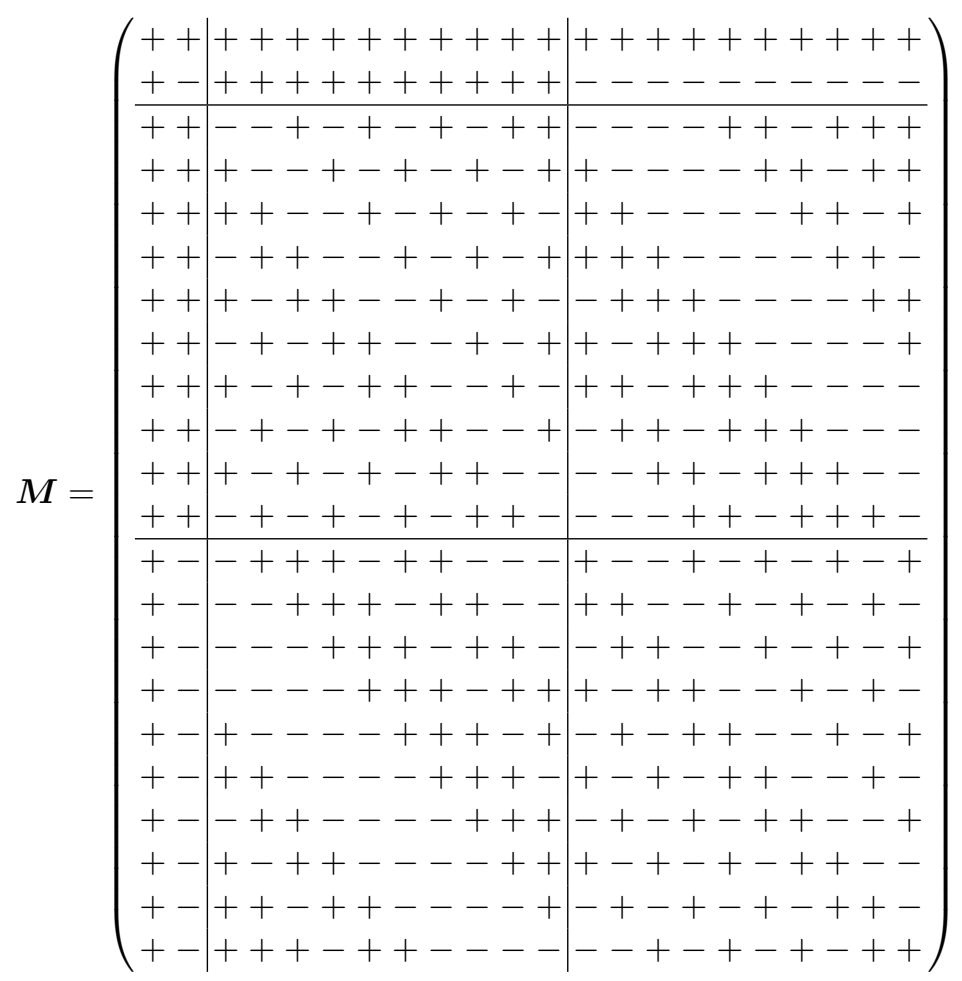

Theorem 1. If $\boldsymbol{A}$ is the incidence matrix of a $\left(v, k ; \lambda_{1}, \ldots, \lambda_{\lfloor v / 2\rfloor}\right) G D S$, then $\boldsymbol{A} \boldsymbol{A}^{\prime}$ is a $v \times v$ circulant matrix with entries

$$
a_{i, j}= \begin{cases}v & \text { if } i=j \\ v-4 k+8 \lambda_{v / 2} & \text { if }|j-i|=\frac{v}{2} \text { and } v \text { is even } \\ v-4 k+4 \lambda_{s} & \text { otherwise, }\end{cases}
$$

where $s=\min \{|j-i|,|v-(j-i)|\}$. Furthermore, $\boldsymbol{A} \boldsymbol{A}^{\prime}=\boldsymbol{A}^{\prime} \boldsymbol{A}$.

Back circulant matrices do not commute, but circulant matrices do. Since every incidence matrix of a general difference set is circulant, we have the following.

Lemma 3. If $\boldsymbol{A}$ and $\boldsymbol{B}$ are the incidence matrices of two general difference sets, then $\boldsymbol{A} \boldsymbol{B}^{\prime}=\boldsymbol{B}^{\prime} \boldsymbol{A}$.

Next, using Theorem 1 and Lemma 3, matrices $\boldsymbol{P}, \boldsymbol{P}_{I}$, and $\boldsymbol{P}_{I I}$ are constructed via GSDSs.

Lemma 4. Let $\boldsymbol{A}, \boldsymbol{B}$ be the incidence matrices of two base blocks of a 2 $\left\{2 t ; k_{1}, k_{2} ; \lambda_{1}, \lambda_{2}, \ldots, \lambda_{t}\right\} G S D S$. 
(a) If $k_{1}=k_{2}=t, \lambda_{t}=\lfloor t / 2\rfloor, \lambda_{i}=t$ or $t-1$ such that $n(t)=\lfloor(t-1) / 2\rfloor$ and $n(t-1)=\lceil(t-1) / 2\rceil$, then $2 \boldsymbol{J}_{2 t}+\boldsymbol{A} \boldsymbol{A}^{\prime}+\boldsymbol{B} \boldsymbol{B}^{\prime}=\boldsymbol{P}$.

(b) If $k_{1}=k_{2}=t, \lambda_{t}=\lfloor t / 2\rfloor$ and $\lambda_{i}=t-\left(\left(1+(-1)^{i+1}\right) / 2\right)$ for all $1 \leq i \leq t-1$, then $2 \boldsymbol{J}_{2 t}+\boldsymbol{A} \boldsymbol{A}^{\prime}+\boldsymbol{B} \boldsymbol{B}^{\prime}=\boldsymbol{P}_{I}$.

(c) If $k_{1}=t, k_{2}=t+1, \lambda_{t}=(t+1) / 2$ and $\lambda_{i}=t+\left(\left(1+(-1)^{i}\right) / 2\right)$ for all $1 \leq i \leq t-1$, then $2 \boldsymbol{J}_{2 t}+\boldsymbol{A} \boldsymbol{A}^{\prime}+\boldsymbol{B} \boldsymbol{B}^{\prime}=\boldsymbol{P}_{I I}$.

Notice that $\lambda_{t}$ is not equal to $\lfloor t / 2\rfloor$ in $(c)$. Since the total number of $\lambda$ should equal the total number of pairs in a GSDS, it follows that $\sum_{i=1}^{t} \lambda_{i}=\left(\begin{array}{c}k_{1} \\ 2\end{array}\right)+\left(\begin{array}{c}k_{2} \\ 2\end{array}\right)$. When $t$ is even, $\lambda_{t}$ in $(c)$ is $t / 2$ by Theorem 1 . However, $\sum_{i=1}^{t} \lambda_{i}=(2 t+1)(t / 2-$ 1) $+t+\lambda_{t}=t^{2}-1$ is not equal to $\left(\begin{array}{c}t \\ 2\end{array}\right)+\left(\begin{array}{c}t+1 \\ 2\end{array}\right)=t^{2}$. Hence, $\boldsymbol{P}_{I I}$ does not exist when $t$ is even. By Lemma 3 and Lemma 4 , near-Hadamard designs are provided by the following.

Theorem 2. Let $n=4 t+2$ and $\boldsymbol{M}$ be a matrix constructed by a $2-\left\{2 t ; k_{1}, k_{2} ; \lambda_{1}\right.$, $\left.\lambda_{2}, \ldots, \lambda_{t}\right\} G S D S$.

(a) If $k_{1}=k_{2}=t, \lambda_{t}=\lfloor t / 2\rfloor, \lambda_{i}=t$ or $t-1$ such that $n(t)=\lfloor(t-1) / 2\rfloor$ and $n(t-1)=\lceil(t-1) / 2\rceil$, then $\boldsymbol{M}$ is a near-Hadamard design.

(b) If $k_{1}=k_{2}=t, \lambda_{t}=\lfloor t / 2\rfloor$ and $\lambda_{i}=t-\left(\left(1+(-1)^{i+1}\right) / 2\right)$ for all $1 \leq i \leq t-1$, then $\boldsymbol{M}$ is a near-Hadamard design of Type $I$.

(c) If $k_{1}=t, k_{2}=t+1, \lambda_{t}=(t+1) / 2$ and $\lambda_{i}=t+\left(\left(1+(-1)^{i}\right) / 2\right)$ for all $1 \leq i \leq t-1$, then $\boldsymbol{M}$ is a near-Hadamard design of Type $I I$.

Cohn (2000) found a design of order 22 which has 0.90 efficiency, where his efficiency is defined to be the ratio $|\boldsymbol{D}| /\left|\boldsymbol{D}_{o}\right|$ and $\left|\boldsymbol{D}_{o}\right|$ is the Ehlich's upper bound. Álvarez et al. (2012) proposed two algorithms and found designs of order 22 and 34 with 0.90 efficiency. More bounds for the maximum determinant were discussed by Koukouvinos, Mitrouli, and Seberry (20001).

Table 1 of the supplementary material provides a list of all $2-\left\{2 t ; k_{1}, k_{2} ; \lambda_{1}\right.$, $\left.\lambda_{2}, \ldots, \lambda_{t}\right\}$ GSDSs satisfying Theorem 2 when $1 \leq t \leq 25$. The third column reports the types of near-Hadamard designs, where Type $G$ means general form. When $n=22,34$, and 70, a near-Hadamard design of Type $I$ is found. Since Type $I$ may not exist when $n=78$ and 94 Orrick and Slolmon (2002), but Type $I I$ design was found in both cases. An exhaustive search shows that when $n=58$, Types $I$ and $I I$ do not exist, so the best among all possible near-Hadamard designs of order 58 is listed in the table. Furthermore, each of the near-Hadamard designs of order $22,34,58,70,78$, and 94 constructed by the GSDS has at least 99.5\% $D$-efficiency.

Corollary 1. A GSDS-constructed near-Hadamard design of order n exists whenever $6 \leq n \leq 98$. 
We conjecture that the near-Hadamard designs of Type $I$ and $I I$ for $n=$ $22,34,70,78$, and 94 are $D$-optimal.

\section{Design Table}

Table 2 provides lower bounds for the $D$-efficiencies of GSDS-based nearHadamard designs of orders $n \equiv 2(\bmod 4)$. To calculate these lower bounds, the Ehlich's upper bound was used.

\section{Discussion and Conclusion}

By the definition of supplementary difference sets (Wallis ([प/2)), SDS is a special case of GSDS where all $\lambda$ 's are the same. Fletcher, Gysin, and Seberry (200I) constructed Hadamard designs with two circulant cores via SDSs. This is a unified method for constructing Hadamard designs. Kotsireas, Koukouvinos, and Seberry (2006) provided a series of methods to construct Hadamard designs with two circulant cores via SDSs, and conjectured such Hadamard designs of order $2 l+2$ exist for any odd $l \geq 3$. Following our notation, the matrix used by Fletcher, Gysin, and Seberry (2001) is matrix (ए.D) with $c=-1$. The existence of a $2-\{2 t-1 ; t-1 ; t-2\}$ GSDS implies the existence of a Hadamard design of order $4 t$, and $2-\{2 t-1 ; t-1 ; t-2\}$ GSDS is conjectured to exists for every $t \geq 2$. Chiarandini et al. (2008) proposed heuristic algorithms for constructing Hadamard designs with two circulant cores via SDSs. Our contribution here is making the connection between GSDSs and near-Hadamard designs of Type $I$ and Type II. Algorithms for searching GSDSs that exploit this connection will be considered in our future work.

We introduced a class of designs called the near-Hadamard designs of order $4 t+2$. To our best knowledge, there is no prior method for the construction of almost $D$-optimal designs of any order $n$ in which the Ehlich's upper bound is not achievable. We proposed general supplementary difference sets (GSDSs) as a unified method to directly construct near-Hadamard designs.

We derive a formula for the determinants of these designs (both Type $I$ and $I I)$, and provide a list of GSDSs for constructing almost $D$-optimal designs of orders 22,34,58,70,78, and 94. Our newly found Type $I$ design of order 22 has the same determinant as that of the order 22 design listed on http://www. indiana.edu/ maxdet, yet the two designs are not equivalent. This answers the uniqueness questions posed there in the negative.

The GSDS method greatly reduces the search space, to at most $\left(\begin{array}{c}2 t-1 \\ t-1\end{array}\right)\left(\begin{array}{c}2 t-1 \\ t-1\end{array}\right)$

combinations when $t=n / 4$ and less than $\left(\begin{array}{c}2 t \\ t\end{array}\right)\left(\begin{array}{c}2 t \\ t+1\end{array}\right)$ when $t=(n-2) / 4$. The method provides various criteria for further reducing the search space efficiently. Without requiring determinants, Theorem 2 provides helpful criteria for finding Type I, Type II near-Hadamard designs and near-Hadamard designs in general. 
Table 2. Lower Bounds on D-efficiencies of GSDS-based (near)-Hadamard designs.

\begin{tabular}{|cccccc|}
\hline$t$ & $N=4 t+2$ Runs & $D$-efficiencies & $t$ & $N=4 t+2$ Runs & $D$-efficiencies \\
\hline 1 & 6 & $92.83 \%$ & 2 & 10 & $97.67 \%$ \\
\hline 3 & 14 & $97.79 \%$ & 4 & 18 & $99.34 \%$ \\
\hline 5 & 22 & $99.56 \%$ & 6 & 26 & $99.10 \%$ \\
\hline 7 & 30 & $98.89 \%$ & 8 & 34 & $99.82 \%$ \\
\hline 9 & 38 & $99.16 \%$ & 10 & 42 & $99.88 \%$ \\
\hline 11 & 46 & $99.36 \%$ & 12 & 50 & $99.62 \%$ \\
\hline 13 & 54 & $99.93 \%$ & 14 & 58 & $99.50 \%$ \\
\hline 15 & 62 & $99.38 \%$ & 16 & 66 & $99.43 \%$ \\
\hline 17 & 70 & $99.50 \%$ & 18 & 74 & $99.50 \%$ \\
\hline 19 & 78 & $99.91 \%$ & 20 & 82 & $99.73 \%$ \\
\hline 21 & 86 & $99.69 \%$ & 22 & 90 & $99.61 \%$ \\
\hline 23 & 94 & $99.93 \%$ & 24 & 98 & $99.60 \%$ \\
\hline
\end{tabular}

These criteria are based on frequencies of differences. Hence, checking these criteria is much cheaper than computing determinants of candidate designs. This makes it possible to design efficient search algorithms for finding near-Hadamard designs. By making good use of these criteria many unsuitable sequences can be ignored. For instance, a $2-\{34 ; 17,17 ; 16,17, \ldots, 16,17,8\}$ GSDS provides a Type $I$ near-Hadamard matrix of order 70 . If one of the two base blocks is $\{1,3,5, \ldots, 33\}$, then it is a $(34,17 ; 0,17, \ldots, 0,17,0)$ GDS. By definition, another base block must be a $(34,17 ; 16,0, \ldots, 16,0,8)$ GDS. However, for any three integers in $Z_{34}$ there must exist two integers such that their difference is even. Hence it is impossible to find a $(34,17 ; 16,0, \ldots, 16,0,8)$ GDS, and the set $\{1,3,5, \ldots, 33\}$ can be ruled out. GSDS based search algorithms that exploit the Theorem 2 criteria for finding Type $I$ and Type $I I$ designs for large $n$ will be a topic of future research.

Table 2 shows the $D$-efficiencies of our GSDS-based near-Hadamard designs. When $2 n-2$ is not a sum of two squares, Type $I$ and $I I$ near-Hadamard designs are conjectured to be $D$-optimal. Table 1 in the supplementary materials provides the corresponding catalog.

\section{Acknowledgement}

The authors deeply appreciate the careful corrections and constructive suggestions of an associate editor and two reviewers. This work was supported by a Career Development Award of Academia Sinica (Taiwan) grant 103-CDA-M04 for Phoa, and a Ministry of Science and Technology (Taiwan) grant 102-2628-M001-002-MY3 for Phoa and Lin. 


\section{Appendix}

Proof of Lemma 2. If $\boldsymbol{A}_{\alpha \times \alpha}=\operatorname{cric}(x, y, y, \ldots, y)$, then $\operatorname{det}(\boldsymbol{A})=(x+(\alpha-$ 1) $y)(x-y)^{\alpha-1}$. It is easy to see this by adding each column to the first column. (i) Subtract $c_{1} / n$ times the first row of $\boldsymbol{M}_{I} \boldsymbol{M}_{I}^{\prime}$ from each row starting with $c_{1}$, and then $c_{1} / n$ times the first column from each column starting with $c_{1}$. Similarly, remove each element $c_{2}$ in the second row and column. Without loss of generality, we may assume that $c_{1}=c_{2}=2$. Without altering the determinant, this changes $\boldsymbol{M}_{I} \boldsymbol{M}_{I}^{\prime}$ to

$$
\boldsymbol{H}=\left|\begin{array}{llll}
n & 0 & 0 & 0 \\
0 & n & 0 & 0 \\
0 & 0 & \boldsymbol{Q} & 0 \\
0 & 0 & 0 & \boldsymbol{Q}
\end{array}\right|
$$

where $\boldsymbol{Q}=\operatorname{circ}(N, a, b, \ldots, a, b, a)$ is a square matrix of order $(n-2) / 2$. The elements $N, a$, and $b$ are $n-(4 / n),(-2)-(4 / n)$ and $2-(4 / n)$, respectively. Now, we calculate $\operatorname{det}(\boldsymbol{Q})$. After adding each column to the first column and removing a factor $(N+((n-2) / 4) a+((n-6) / 4) b)$ of the first column, the first column becomes the all-ones vector. Subtracting the first row from every other row, using the properties of the determinant and the cofactor formula, we get $\operatorname{det}(\boldsymbol{Q})=(N+((n-2) / 4) a+((n-6) / 4) b)(N-b)^{(n-6) / 4} \cdot \operatorname{det}(\boldsymbol{W})$, where $\boldsymbol{W}=\operatorname{circ}(N-a, b-a, \ldots, b-a)$ is a square matrix of order $(n-2) / 4$. Using $\operatorname{det}(\boldsymbol{W})=((N-a)+(b-a)(n-6) / 4)(N-b)^{(n-6) / 4}$, and simplifying, $\operatorname{det}\left(\boldsymbol{M}_{I} \boldsymbol{M}_{I}^{\prime}\right)=\operatorname{det}(\boldsymbol{H})=n^{2} \cdot[\operatorname{det}(\boldsymbol{Q})]^{2}=4(n-2)^{n}$. Therefore, $\operatorname{det}\left(\boldsymbol{M}_{I}\right)=$ $2(n-2)^{n / 2}$.

(ii) Similarly, reduce $\boldsymbol{M}_{I I} \boldsymbol{M}_{I I}^{\prime}$ to a matrix that has the form of $\boldsymbol{H}$ without altering its determinant. Now $\boldsymbol{Q}$ is a circulant matrix of order $(n-2) / 2$ with the first row $(N, a, b, \ldots, a, b, b, b, a, b, \ldots, a, b, a)$, where $N=n-(4 / n), a=$ $(-2)-(4 / n)$ and $b=2-(4 / n)$. Observe that

$$
\boldsymbol{Q}=\left(\begin{array}{ll}
\boldsymbol{Q}_{1} & \boldsymbol{Q}_{2} \\
\boldsymbol{Q}_{2} & \boldsymbol{Q}_{1}
\end{array}\right), \text { where } \boldsymbol{Q}_{1}=\left(\begin{array}{cccccccc}
N & a & b & a & b & \cdots & a & b \\
a & N & a & b & a & \cdots & b & a \\
b & a & N & a & b & \cdots & a & b \\
a & b & a & N & a & \cdots & b & a \\
b & a & b & a & N & \cdots & a & b \\
\vdots & \vdots & \vdots & \vdots & \vdots & \ddots & \vdots & \vdots \\
a & b & a & b & a & \cdots & N & a \\
b & a & b & a & b & \cdots & a & N
\end{array}\right), \text { and }
$$




$$
\boldsymbol{Q}_{2}=\left(\begin{array}{cccccccc}
b & b & a & b & a & \cdots & b & a \\
b & b & b & a & b & \cdots & a & b \\
a & b & b & b & a & \cdots & b & a \\
b & a & b & b & b & \cdots & a & b \\
a & b & a & b & b & \cdots & b & a \\
\vdots & \vdots & \vdots & \vdots & \vdots & \ddots & \vdots & \vdots \\
b & a & b & a & b & \cdots & b & b \\
a & b & a & b & a & \cdots & b & b
\end{array}\right) \text {, where } \boldsymbol{Q}_{1} \text { and } \boldsymbol{Q}_{2} \text { are } \frac{n-2}{4} \times \frac{n-2}{4} .
$$

Since $\operatorname{det}(\boldsymbol{Q})=\operatorname{det}\left(\boldsymbol{Q}_{1}-\boldsymbol{Q}_{2}\right) \cdot \operatorname{det}\left(\boldsymbol{Q}_{1}+\boldsymbol{Q}_{2}\right)$, we evaluate $\operatorname{det}\left(\boldsymbol{Q}_{1}-\boldsymbol{Q}_{2}\right)$ first. Let $N^{\prime}=N-b, a^{\prime}=a-b$ and $b^{\prime}=b-a$. Adding each column except the 1st to the 2nd column, then interchanging the first two rows and columns, we get

$$
\begin{aligned}
& \operatorname{det}\left(\boldsymbol{Q}_{1}-\boldsymbol{Q}_{2}\right)=\left|\begin{array}{ccccccc}
N^{\prime}+a^{\prime} & a^{\prime} & a^{\prime} & b^{\prime} & \cdots & b^{\prime} & a^{\prime} \\
0 & N^{\prime} & b^{\prime} & a^{\prime} & \cdots & a^{\prime} & b^{\prime} \\
N^{\prime}+a^{\prime} & b^{\prime} & N^{\prime} & a^{\prime} & \cdots & a^{\prime} & b^{\prime} \\
N^{\prime}+a^{\prime} & a^{\prime} & a^{\prime} & N^{\prime} & \cdots & b^{\prime} & a^{\prime} \\
\vdots & \vdots & \vdots & \vdots & \ddots & \vdots & \vdots \\
N^{\prime}+a^{\prime} & a^{\prime} & a^{\prime} & b^{\prime} & \cdots & N^{\prime} & a^{\prime} \\
N^{\prime}+a^{\prime} & b^{\prime} & b^{\prime} & a^{\prime} & \cdots & a^{\prime} & N^{\prime}
\end{array}\right| \\
& =\left|\begin{array}{ccccccc}
N^{\prime}+a^{\prime} & a^{\prime} & a^{\prime} & b^{\prime} & \cdots & b^{\prime} & a^{\prime} \\
0 & N^{\prime} & b^{\prime} & a^{\prime} & \cdots & a^{\prime} & b^{\prime} \\
0 & b^{\prime}-a^{\prime} & N^{\prime}-a^{\prime} & a^{\prime}-b^{\prime} & \cdots & a^{\prime}-b^{\prime} & b^{\prime}-a^{\prime} \\
0 & 0 & 0 & N^{\prime}-b^{\prime} & \cdots & 0 & 0 \\
\vdots & \vdots & \vdots & \vdots & \ddots & \vdots & \vdots \\
0 & 0 & 0 & 0 & \cdots & N^{\prime}-b^{\prime} & 0 \\
0 & b^{\prime}-a^{\prime} & b^{\prime}-a^{\prime} & a^{\prime}-b^{\prime} & \cdots & a^{\prime}-b^{\prime} & N^{\prime}-a^{\prime}
\end{array}\right|
\end{aligned}
$$

Notice that $a^{\prime}+b^{\prime}=0, b^{\prime}-a^{\prime}=8, N^{\prime}-a^{\prime}=n+2, N^{\prime}+a^{\prime}=n-6=N^{\prime}-b^{\prime}$, $N-b=N^{\prime}=n-2, a^{\prime}=-4$, and $b^{\prime}=4$. By using the properties of the determinant and the cofactor formula, $\operatorname{det}\left(\boldsymbol{Q}_{1}-\boldsymbol{Q}_{2}\right)=(n-6)^{(n-6) / 8} \cdot \operatorname{det}(\boldsymbol{E})$, where

$$
\operatorname{det}(\boldsymbol{E})=\left|\begin{array}{cc}
n-2 & 4 \boldsymbol{j} \\
8 \boldsymbol{j} & \boldsymbol{E}^{\prime}
\end{array}\right|, \text { and } \boldsymbol{E}^{\prime}=\operatorname{circ}(n+2,8, \ldots, 8) .
$$

Here, $\boldsymbol{E}^{\prime}$ is a square matrix of order $(n-6) / 8$. Subtracting $8 /(n-2)$ times the first row from every other row yields $\operatorname{det}(\boldsymbol{E})=\left[\left(n^{2}-36\right)+(n-14)(n-6)\right](n-6)^{(n-14) / 8}$. Therefore, $\operatorname{det}\left(\boldsymbol{Q}_{1}-\boldsymbol{Q}_{2}\right)=2(n-4)(n-6)^{(n-6) / 4}$. Now, it is easy to see that $\boldsymbol{Q}_{1}+\boldsymbol{Q}_{2}=\operatorname{circ}(N+b, a+b, \ldots, a+b)$, where $N=n-(4 / n), a=-2-(4 / n)$ and $b=2-(4 / n)$. So $\operatorname{det}\left(\boldsymbol{Q}_{1}+\boldsymbol{Q}_{2}\right)=[(N+b)+((n-6) / 4)(a+b)](N-a)^{(n-6) / 4}=$ $(n+(4 / n))(n+2)^{(n-6) / 4}$. Since $\operatorname{det}\left(\boldsymbol{M}_{I I} \boldsymbol{M}_{I I}^{\prime}\right)=\operatorname{det}(\boldsymbol{H})=n^{2}[\operatorname{det}(\boldsymbol{Q})]^{2}$, we get 
$\operatorname{det}\left(\boldsymbol{M}_{I I}\right)=n \cdot \operatorname{det}(\boldsymbol{Q})=n \cdot \operatorname{det}\left(\boldsymbol{Q}_{1}-\boldsymbol{Q}_{2}\right) \cdot \operatorname{det}\left(\boldsymbol{Q}_{1}+\boldsymbol{Q}_{2}\right)=2(n-4)\left(n^{2}+4\right)(n-$ $6)^{(n-6) / 4}(n+2)^{(n-6) / 4}$.

Proof of Lemma 4. Let $D_{1}$ and $D_{2}$ be two base blocks of a $2-\left\{2 t ; k_{1}, k_{2} ; \lambda_{1}\right.$, $\left.\lambda_{2}, \ldots, \lambda_{t}\right\}$ GSDS, where $D_{1}$ is a $\left(2 t, k_{1} ; \mu_{1}, \mu_{2}, \ldots, \mu_{t}\right)$ GDS, $D_{2}$ is a $\left(2 t, k_{2} ; \mu_{1}^{\prime}\right.$, $\left.\mu_{2}^{\prime}, \ldots, \mu_{t}^{\prime}\right)$ GDS, and $\mu_{i}+\mu_{i}^{\prime}=\lambda_{i}$ for $i=1,2, \ldots, t$. Let $\boldsymbol{A} \boldsymbol{A}^{\prime}=\left(a_{i, j}\right)$ and $\boldsymbol{B}^{\prime}=\left(b_{i, j}\right)$.

(a) Let $k_{1}=k_{2}=t, \lambda_{t}=\lfloor t / 2\rfloor, \lambda_{i}=t$ or $t-1$ such that $n(t)=\lfloor(t-1) / 2\rfloor$ and $n(t-1)=\lceil(t-1) / 2\rceil$. By Theorem $1, a_{i, i}+b_{i, i}=4 t$. If $|j-i| \neq t$ then $a_{i, j}+b_{i, j}=2(2 t)-4(2 t)+4\left(\mu_{i}+\mu_{i}^{\prime}\right)=0$ or -4 when $\lambda_{i}=t$ or $t-1$. If $|j-i|=t$ then $a_{i, j}+b_{i, j}=2(2 t)-4(2 t)+8\left(\mu_{t}+\mu_{t}^{\prime}\right)=4 t-8 t+8(\lfloor t / 2\rfloor)=-4$ or 0 when $t$ is odd or even. It follows that $2 \boldsymbol{J}_{2 t}+\boldsymbol{A} \boldsymbol{A}^{\prime}+\boldsymbol{B B}^{\prime}=\boldsymbol{P}$.

(b) Let $k_{1}=k_{2}=t, \lambda_{t}=\lfloor t / 2\rfloor$ and $\lambda_{i}=t-\left(\left(1+(-1)^{i+1}\right) / 2\right)$ for all $1 \leq i \leq t-1$. By Theorem 1, $a_{i, i}+b_{i, i}=4 t$. If $|j-i| \neq t$ then $a_{i, j}+b_{i, j}=2(2 t)-4(2 t)+$ $4\left(\mu_{i}+\mu_{i}^{\prime}\right)=4 t-8 t+4\left(t-\left(\left(1+(-1)^{i+1}\right) / 2\right)\right)=-4$ or 0 when $i$ is odd or even. If $|j-i|=t$ then $a_{i, j}+b_{i, j}=2(2 t)-4(2 t)+8\left(\mu_{t}+\mu_{t}^{\prime}\right)=4 t-8 t+8(\lfloor t / 2\rfloor)=-4$ or 0 when $t$ is odd or even. Therefore, $2 \boldsymbol{J}_{2 t}+\boldsymbol{A} \boldsymbol{A}^{\prime}+\boldsymbol{B} \boldsymbol{B}^{\prime}=\boldsymbol{P}_{I}$.

(c) Let $k_{1}=t, k_{2}=t+1, \lambda_{t}=(t+1) / 2$ and $\lambda_{i}=t+\left(\left(1+(-1)^{i}\right) / 2\right)$ for all $1 \leq i \leq t-1$. By Theorem $1, a_{i, i}+b_{i, i}=4 t$. If $|j-i| \neq t$ then $a_{i, j}+b_{i, j}=$ $2(2 t)-4(2 t+1)+4\left(\mu_{i}+\mu_{i}^{\prime}\right)=4 t-8 t-4+4\left(t+\left(\left(1+(-1)^{i}\right) / 2\right)\right)=-4$ or 0 when $i$ is odd or even. If $|j-i|=t$ then $a_{i, j}+b_{i, j}=2(2 t)-4(2 t+1)+8\left(\mu_{t}+\mu_{t}^{\prime}\right)=$ $4 t-8 t+8((t+1) / 2)=0$ when $t$ is odd. Hence, $2 \boldsymbol{J}_{2 t}+\boldsymbol{A} \boldsymbol{A}^{\prime}+\boldsymbol{B} \boldsymbol{B}^{\prime}=\boldsymbol{P}_{I I}$.

Proof of Theorem 2. Let $\boldsymbol{A}$ and $\boldsymbol{B}$ be the incidence matrices of two base blocks of a $2-\left\{2 t ; k_{1}, k_{2} ; \lambda_{1}, \lambda_{2}, \ldots, \lambda_{t}\right\}$ GSDS. Using $\boldsymbol{A}$ and $\boldsymbol{B}$ to construct the $n \times n$ matrix $\boldsymbol{M}$ defined in (B.]), we have

$$
\begin{aligned}
& \boldsymbol{M} \boldsymbol{M}^{\prime}= \\
& \left(\begin{array}{rrrr}
n & 0 & 2 c \boldsymbol{j}^{\prime}+\boldsymbol{j}^{\prime} \boldsymbol{A}+\boldsymbol{j}^{\prime} \boldsymbol{B}^{\prime} & \boldsymbol{j}^{\prime} \boldsymbol{B}-\boldsymbol{j}^{\prime} \boldsymbol{A}^{\prime} \\
0 & n & \boldsymbol{j}^{\prime} \boldsymbol{A}-\boldsymbol{j}^{\prime} \boldsymbol{B}^{\prime} & 2 c \boldsymbol{j}^{\prime}+\boldsymbol{j}^{\prime} \boldsymbol{B}+\boldsymbol{j}^{\prime} \boldsymbol{A}^{\prime} \\
2 c \boldsymbol{j}+\boldsymbol{A}^{\prime} \boldsymbol{j}+\boldsymbol{B} \boldsymbol{j} & \boldsymbol{A}^{\prime} \boldsymbol{j}-\boldsymbol{B} \boldsymbol{j} & 2 \boldsymbol{J}_{2 t}+\boldsymbol{A}^{\prime} \boldsymbol{A}+\boldsymbol{B} \boldsymbol{B}^{\prime} & \boldsymbol{A}^{\prime} \boldsymbol{B}-\boldsymbol{B} \boldsymbol{A}^{\prime} \\
\boldsymbol{B}^{\prime} \boldsymbol{j}-\boldsymbol{A} \boldsymbol{j} & 2 c \boldsymbol{j}+\boldsymbol{B}^{\prime} \boldsymbol{j}+\boldsymbol{A} \boldsymbol{j} & \boldsymbol{B}^{\prime} \boldsymbol{A}-\boldsymbol{A} \boldsymbol{B}^{\prime} & 2 \boldsymbol{J}_{2 t}+\boldsymbol{B}^{\prime} \boldsymbol{B}+\boldsymbol{A} \boldsymbol{A}^{\prime}
\end{array}\right)
\end{aligned}
$$

We prove (a) first. Since $\boldsymbol{A}$ and $\boldsymbol{B}$ are circulant matrices corresponding to the base block with size $t$, there should be $t$ " -1 " and $t$ " +1 " in each column and each row. Therefore, the row sum and column sum of $\boldsymbol{A}$ and $\boldsymbol{B}$ are zero, this implies that $2 c \boldsymbol{j}^{\prime}+\boldsymbol{j}^{\prime} \boldsymbol{A}+\boldsymbol{j}^{\prime} \boldsymbol{B}^{\prime}=2 c \boldsymbol{j}^{\prime}, 2 c \boldsymbol{j}+\boldsymbol{A}^{\prime} \boldsymbol{j}+\boldsymbol{B} \boldsymbol{j}=2 c \boldsymbol{j}$ and $\boldsymbol{j}^{\prime} \boldsymbol{A}-\boldsymbol{j}^{\prime} \boldsymbol{B}^{\prime}=\boldsymbol{j}^{\prime} \boldsymbol{B}-\boldsymbol{j}^{\prime} \boldsymbol{A}^{\prime}=\mathbf{0}$. By Lemma $3, \boldsymbol{B}^{\prime} \boldsymbol{A}-\boldsymbol{A} \boldsymbol{B}^{\prime}=\boldsymbol{A}^{\prime} \boldsymbol{B}-\boldsymbol{B} \boldsymbol{A}^{\prime}=\mathbf{0}$. By Lemma $4,2 \boldsymbol{J}_{2 t}+\boldsymbol{A}^{\prime} \boldsymbol{A}+\boldsymbol{B} \boldsymbol{B}^{\prime}=\boldsymbol{P}$. Hence, $\boldsymbol{M}$ is a near-Hadamard design. Similarly, if $\lambda_{i}$ satisfies (b) then, by Lemma 4 , all submatrices except the diagonals are as in the proof of part $(a)$ and $2 \boldsymbol{J}_{2 t}+\boldsymbol{A}^{\prime} \boldsymbol{A}+\boldsymbol{B} \boldsymbol{B}^{\prime}=\boldsymbol{P}_{I}$. Therefore, $\boldsymbol{M}$ is a 
near-Hadamard design of Type $I$. If $k_{1}=t, k_{2}=t+1$ and $\lambda_{i}$ satisfies $(c)$, then set $c=1$. It follows that $2 \boldsymbol{j}^{\prime}+\boldsymbol{j}^{\prime} \boldsymbol{A}+\boldsymbol{j}^{\prime} \boldsymbol{B}^{\prime}=2 \boldsymbol{j}+\boldsymbol{A}^{\prime} \boldsymbol{j}+\boldsymbol{B} \boldsymbol{j}=\mathbf{0}, \boldsymbol{j}^{\prime} \boldsymbol{A}-\boldsymbol{j}^{\prime} \boldsymbol{B}^{\prime}=2 \boldsymbol{j}^{\prime}$, and $\boldsymbol{j}^{\prime} \boldsymbol{B}-\boldsymbol{j}^{\prime} \boldsymbol{A}^{\prime}=-2 \boldsymbol{j}^{\prime}$. By Lemma 4 , we have $2 \boldsymbol{J}_{2 t}+\boldsymbol{A}^{\prime} \boldsymbol{A}+\boldsymbol{B} \boldsymbol{B}^{\prime}=\boldsymbol{P}_{I I}$. Hence $\boldsymbol{M}$ is a near-Hadamard design of Type $I I$.

\section{References}

Álvarez, V., Armario, J. A., Frau, M. D. and Gudiel, F. (2012). The maximal determinant of cocyclic $(-1,1)$-matrices over $D_{2 t}$. Linear Algebra and its Applications 436(4), 858-873.

Andersen, I. (1990). Combinatorial Designs: Construction Methods. Ellis Horwood, Chichester and New York.

Bose, R. C. (1939). On the construction of balanced incomplete block designs. Ann. Eugenics $\mathbf{9 ( 4 ) , 3 5 3 - 3 9 9 . ~}$

Chiarandini, M., Kotsireas, I. S., Koukouvinos, C. and Paquete, L. (2008). Heuristic algorithms for Hadamard matrices with two circulant cores. Theoret. Computer Sci. 407, 274-277.

Cohn, J. H. E. (1989). On determinants with elements \pm 1 , II. Bull. London Math. Soc. 21, $36-42$.

Cohn, J. H. E. (2000). Almost D-optimal designs. Utilitas Math. 57, 121-128.

Colbourn, C. J. (2010). CRC Handbook of Combinatorial Designs. CRC press.

Đoković, D. Ž. (1997). Some new D-optimal designs. Austral. J. Combinatorics, 15, 221-232.

Đoković, D. Ž. and Kotsireas, I. S. (2012). New results on D-optimal matrices. J. Combinator. Designs, 20, 278-289.

Ehlich, H. (1964). Determinantenabschätzungen für binäre matrizen. Math. Zeitschrift 83, 123132.

Fletcher, R. J., Gysin, M. and Seberry, J. (2001). Application of the discrete Fourier transform to the search for generalised Legendre pairs and Hadamard matrices. Austral. J. Combinatorics 23, 75-86.

Gysin, M. and Seberry, J. (1998). An experimental search and new combinatorial designs via a generalisation of cyclotomy. J. Combin. Math. Combin. Comput. 27, 143-160.

Hadamard, J. (1893). Résolution d'une question relative aux déterminants. Bull. Sci. Math. 17, 240-246.

Hall, M. and Ryser, H. J. (1951). Cyclic incidence matrices. Canad. J. Math. 3, 95-502.

Jones, B. and Nachtsheim, C. J. (2011). A class of three-level designs for definitive screening in the presence of second-order effects. J. Quality Tech. 43, 1-15.

Kotsireas, I. S., Koukouvinos, C. and Seberry, J. (2006). Hadamard ideas and Hadamard matrices with circulant core. European J.Combinatorics 27, 658-668.

Koukouvinos, C., Kounias, S. and Seberry, J. (1991). Supplementary difference sets and optimal designs. Discrete Math. 88, 49-58.

Koukouvinos, C., Mitrouli, M. and Seberry, J. (2000). Bounds on the maximum determinant for $(1,-1)$ matrices. Bull. Inst. Combin. Appl. 29, 39-48.

Lin, Y.-L., Phoa, F. K. H. and Kao, M.-H. (2015). Partial Hadamard designs: Construction via general difference sets and application to experiments in functional Magnetic Resonance Imaging (fMRI). submitted.

Orrick, W. P. and Solomon, B. (2000). The Hadamard maximal determinant problem. http: //Www. indiana.edu/ maxdet/. Last modified 17 Oct. 2012. 
Phoa, F. K. H. and Lin, D. K. (2015) A systematic approach for the construction of definitive screening designs. Statist. Sinica, 25, 853-861.

Phoa, F. K., Wong, W. K. and Xu, H. (2009). The need of considering the interactions in the analysis of screening designs. J. Chemometrics, 23(10), 545-553.

Phoa, F. K., Xu, H. and Wong, W. K. (2009). The use of nonregular fractional factorial designs in combination toxicity studies. Food and Chemical Toxic. 47, 2183-2188.

Sylvester, J. J. (1867). LX. Thoughts on inverse orthogonal matrices, simultaneous signsuccessions, and tessellated pavements in two or more colours, with applications to Newton's rule, ornamental tile-work, and the theory of numbers. The London, Edinburgh, and Dublin Philosophical Magazine and Journal of Science, 34, 461-475.

Wallis, J. (1972). On supplementary difference sets. Aequationes Math. 8, 242-257.

Wallis, W. D. (2007). Introduction to Combinatorial Designs. CRC Press.

Whiteman, A. L. (1990). A family of D-optimal designs. Ars Combinatoria, 30, 23-26.

Wojtas, M. (1964). On Hadamard's inequality for the determinants of order non-divisible by 4 . Colloquium Math. 1, 73-83.

$\mathrm{Xu}$, H., Phoa, F. K. and Wong, W. K. (2009). Recent developments in nonregular fractional factorial designs. Statist. Surveys 3, 18-46.

Institute of Statistical Science, Academia Sinica, Taipei City 115, Taiwan, R.O.C.

E-mail: gaussla@stat.sinica.edu.tw

Institute of Statistical Science, Academia Sinica, Taipei City 115, Taiwan, R.O.C.

E-mail: fredphoa@stat.sinica.edu.tw

(Received November 2014; accepted March 2015) 